\section{Quand la cataracte conduit à une greffe de la cornée}

Jean-Louis Bourges

> L'œil possède deux lentilles convergentes disposées en série: la cornée et le cristallin. Elles associent leurs puissances. L'image qui est naturellement défocalisée à l'infini, en les traversant successivement, se focalise sur la rétine pour être vue nette (Figure 1). Un œdème peut faire perdre sa transparence à la cornée tandis que le cristallin qui s'opacifie constitue la cataracte. La perte de transparence de l'une ou des deux lentilles affecte la vue significativement. Traiter la cataracte est une pratique banale. Cependant, ceci peut conduire à la perte définitive de transparence de la cornée. II faut alors réaliser une greffe de cette dernière. Comment en vient-on parfois à cela? <

La cataracte est un processus naturel de vieillissement du cristallin, qui s'opacifie et perd son pouvoir d'accommodation avec l'âge. On ne connaît pas actuellement de traitements préventif ou thérapeutique médicaux efficaces. Son traitement est donc actuellement chirurgical. La chirurgie du cristallin consiste à pénétrer dans le globe oculaire pour ôter le cristallin qui est cataracté et le remplacer par une lentille artificielle transparente. Ce faisant, une fragilisation associée de la cornée est alors aggravée par la chirurgie intraoculaire qui expose à la perte de sa transparence. L'évaluation des bénéfices

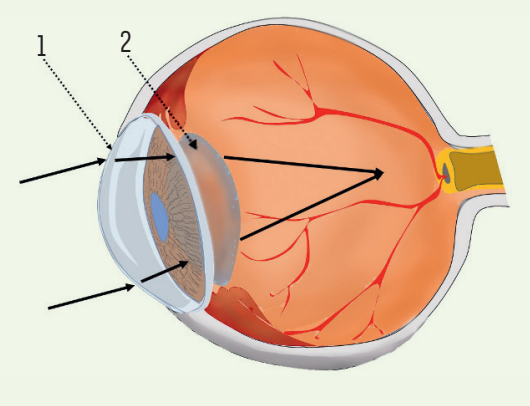

Figure 1. Le globe oculaire focalise l'image (flèches noires pleines) sur son capteur, la rétine, à l'aide de deux lentilles convergentes disposées en série : la cornée (1) puis le cristallin (2).

Vignette (Photo @ Jean-Louis Bourges).

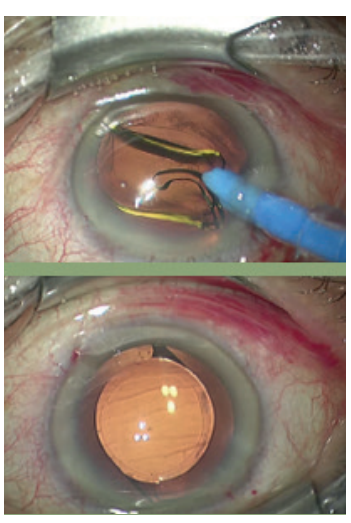

Université de Paris, CRC Inserm 1138-ع17, Assistance publiqueHôpitaux de Paris, Hôpital Cochin, 27 rue du Faubourg Saint-Jacques, 75014 Paris, France.

jean-louis.bourges@aphp.fr

et des risques à intervenir sur une cataracte nécessite donc de connaître l'état préopératoire de la cornée. La stratégie thérapeutique qui est proposée par l'ophtalmologiste en tient compte. II envisage avec son patient, le cas échéant, l'éventualité d'un traitement complémentaire cornéen combiné, simultané ou dans un futur prévisible. En connaissant les bases de la transparence de la cornée comme celles de la chirurgie de la cataracte, il est plus facile de comprendre les solutions actuellement disponibles pour restaurer la fonction des deux lentilles de l'œil.

\section{La cornée et sa transparence}

La cornée est la portion transparente de la paroi de l'œil. Elle possède plusieurs couches différentes sur le plan anatomique. Parmi ces couches, toutes normalement transparentes, le film lacrymal et l'épithélium sont les couches les plus externes au contact de l'environnement. Le stroma cornéen confère à la cornée son épaisseur et sa solidité (Figure 2). II est constitué principalement de fibres de collagène qui sont régulièrement disposées et espacées entre-elles. Pour rester transparent, le stroma cornéen doit être hydraté à un taux constant de 78 \% d'eau, quelles que soient les conditions internes (position debout ou allongée) ou externes (humidité, sècheresse, évaporation) dans lesquelles serait placée la cornée. Ce contrôle de l'hydratation est assuré par la couche cellulaire la plus interne de la cornée : les cellules endothéliales cornéennes (CEC) qui forment l'endothélium cornéen (Figure 2). L'endothélium cornéen est la monocouche cellulaire qui tapisse la face interne de la cornée. II laisse pénétrer l'humeur aqueuse de l'intérieur de l'œil dans la cornée sous les effets conjugués oncotique et pressionnel. L'humeur aqueuse apporte les nutriments nécessaires au fonctionnement des couches cornéennes qui n'ont pas de vaisseaux, transparence oblige. Grâce à un système d'échange ionique, l'endothélium pompe en permanence par effet osmotique l'humeur aqueuse en sens inverse, du stroma vers l'intérieur 


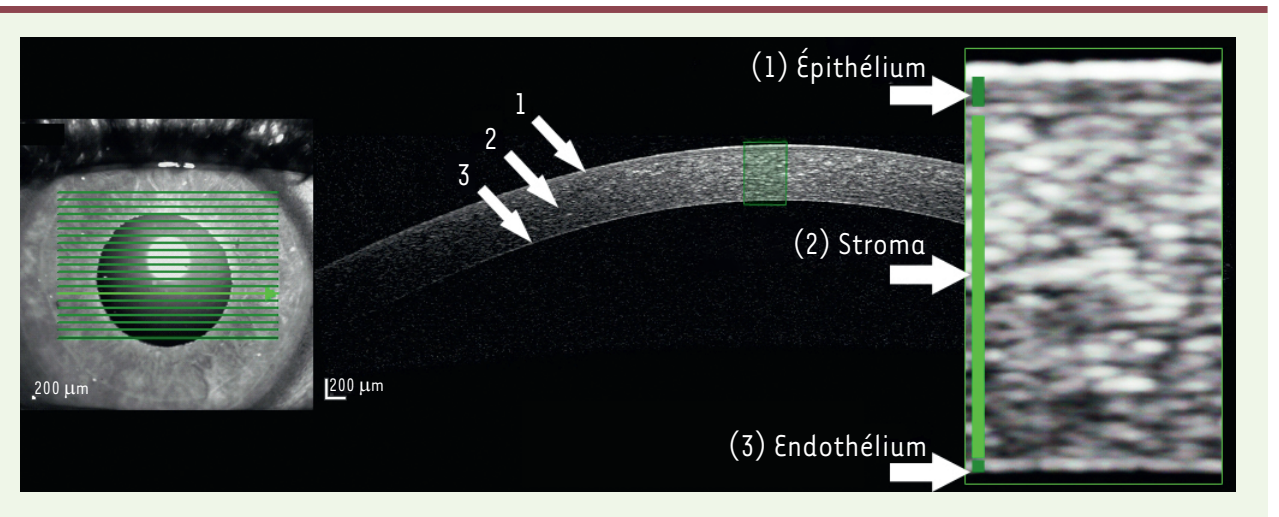

Figure 2. Coupe d'une cornée réalisée avec un dispositif d'imagerie AS-OCT. On distingue l'épithélium en surface (1), le stroma (2) et la couche endothéliale (3) de la cornée.

de l'œil [1]. II assure que I'hydratation du stroma soit constante et homogène. Les lamelles de collagène du stroma gardent ainsi leur espacement régulier. Le stroma cornéen reste donc transparent.

Les CEC, à l'instar des neurones, ne se divisent pas en condition physiologique. Leur cycle mitotique cellulaire est bloqué en phase Gl chez le primate, donc chez l'homme. De ce fait, un individu en possède un capital donné, qui s'étiole progressivement avec le vieillissement. L'homme possède une densité de CદC suffisante pour que celles-ci restent fonctionnelle bien au-delà d'une vie centenaire en l'absence de pathologie intercurrente. Les CEC sont des cellules particulièrement exposées puisque ne se renouvelant pas et étant exposées en perma-

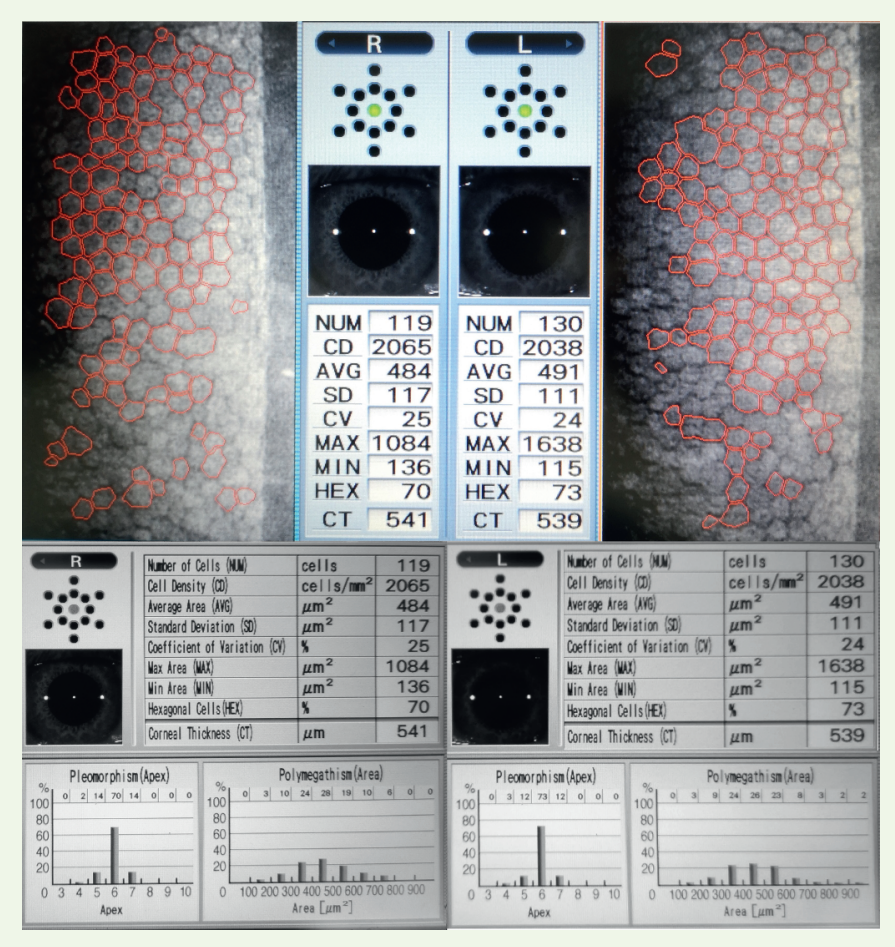

Figure 3. Évaluation de l'endothélium cornéen avec un microscope spéculaire. La forme, le volume, l'hexagonalité et la densité des cellules endothéliales sont évalués quantitativement par analyse d'image automatisée sur plusieurs aires cornéennes grâce à l'image de leur reflet sur la face interne de la cornée. nence aux rayons lumineux et au stress oxydatif qu'ils génèrent. Elles possèdent en conséquence des mécanismes robustes de survie comme un système antioxydant évolué, une résistance à l'apoptose particulière, etc. Lorsqu'une cellule disparaît, les cellules environnantes s'étendent pour maintenir leur confluence et pour que l'endothélium reste fonctionnel.

Il est possible d'explorer directement la morphologie des CદC au travers d'indices quantitatifs tels que la densité de CEC, le coefficient de variation de leur forme, leur volume moyen (Figure 3). Moins l'endothélium contient de CEC, plus leur densité est basse et plus leur volume est grand. La fonction de l'endothélium peut aussi être évaluée en mesurant l'épaisseur de la cornée, qui augmente proportionnellement à l'œdème qu'elle contient. On peut aussi analyser la vue et sa fluctuation nycthémérale ${ }^{1}$.

Lorsque les CEC ne fonctionnent pas convenablement, le stroma cornéen devient œdémateux. La cornée perd sa transparence. La vue de l'œil atteint fluctue dans un premier temps. Elle est altérée, par exemple, le matin au réveil, après le décubitus ${ }^{2}$ prolongé nocturne. Ensuite, l'incompétence des CદC augmentant, l'œdème, et donc l'altération visuelle, perdurent plus longtemps dans la journée, après le décubitus. Des picotements puis une douleur oculaire peuvent s'installer, parfois intenses allant jusqu'à handicaper le sujet.

La dysfonction des CEC peut avoir des origines variées. Toute pathologie atteignant l'endothélium au cours de la vie est susceptible de diminuer le capital des CEC d'une cornée et aboutir, in fine, à son incompétence. Les traumatismes de la cornée, les infections ou les inflammations sont des causes possibles de fragilisation endothéliale. L'implantation de lentille réfractive dans l'œil, proposée typiquement aux forts myopes, est une autre cause possible. Enfin, la cause la plus commune semble être le terrain génétique. Les dysfonction-

Au cours de $24 \mathrm{~h}$.

2 Position couchée. 
nements attribués à quelques gènes comme TCF4 (transcription factor 4), COL 8A2 (collagen 8 alpha2) ou ZEB1 (zinc finger $\varepsilon$-box-binding homeobox 1) ont, par exemple, été identifiés comme responsables d'un dysfonctionnement endothélial chez plus d'une personne sur 200 dans la population générale.

Lorsqu'un endothélium est fragilisé par une affection, la pratique d'une procédure chirurgicale intraoculaire risque de le faire basculer définitivement vers le dysfonctionnement. C'est en particulier le cas de l'intervention intraoculaire la plus fréquente: l'opération de la cataracte.

\section{La cataracte et son traitement}

La cataracte est le durcissement et l'opacification gênante de la lentille cristallinienne, ou cristallin. II s'agit d'un processus naturel de vieillissement qui concernerait plus de 96 millions de personnes dans le monde [2]. Son premier symptôme est la perte de la plasticité cristallinienne qui permet l'accommodation, qui se traduit par l'apparition de la presbytie. Dans les dizaines d'années suivant l'apparition de ce premier symptôme, le cristallin s'opacifie, empêchant l'image de le traverser convenablement. La chirurgie de la cataracte a pour

\section{La phacoémulsification de la cataracte (Figure 4A)}

II n'existe pas actuellement de traitement médical préventif ou curatif efficace de la cataracte. Seule la chirurgie permet sa cure. La phacoémulsification est la technique de référence pour opérer la cataracte en 2020. La phakoexérèse par phacoémulsification se pratique depuis 1962. À cette date, Charles Kelman proposa la technique de phacoémulsification encore utilisée à ce jour, agrémentée de nombreux raffinements supplémentaires pour extraire la cataracte. Elle est effectuée en règle sous anesthésie locale, dans un bloc opératoire, en chirurgie ambulatoire. Son principe consiste à accéder au cristallin à travers une petite incision en périphérie de la cornée (limbique) et au travers d'une pupille dilatée préalablement. Une substance viscoélastique transparente maintient le volume oculaire pendant que l'on pratique une ouverture ronde calibrée (capsulorhexis) sur la face antérieure du sac cristallinien appelé «sac capsulaire ». Après avoir désolidarisé le cristallin de son sac par hydrodissection, on fragmente puis émulsifie le cristallin avec un phacoémulsificateur, sorte d'aiguille biseautée à large diamètre qui aspire les fragments cristalliniens et vibre à une vitesse ultrasonore pour les émulsifier. Le bout de l'instrument infuse un liquide adapté au milieu intraoculaire en quantité contrôlée, identique au volume aspiré. Cela permet de maintenir la pression intraoculaire constante en prévenant l'affaissement aléatoire des volumes intraoculaires. Ensuite, l'intérieur du sac capsulaire est vidé du cortex cristallinien résiduel adhérent, nettoyé puis rempli à nouveau de substance viscoélastique pour l'expandre. Ainsi, il est prêt à recevoir l'implant capsulaire qu'est la lentille intraoculaire artificielle. Cette dernière est introduite dans le sac capsulaire, enroulée dans une cartouche d'injection. Elle s’y déploie dans le viscoélastique qui est ensuite rincé totalement. L'auto-étanchéité de l'incision cornéenne en forme de clapet est vérifiée pour terminer l'intervention. II n'est pas nécessaire de placer un pansement, une coque transparente de sécurité suffit. but d'extraire le cristallin durci et opacifié. Elle prévoie de le remplacer dans sa fonction par une lentille artificielle intraoculaire (LIO). Dans l'idéal, la LIO est placée à l'endroit anatomique du cristallin naturel.

L'intervention de cataracte par phacoémulsification, considérée comme la référence de ce type de chirurgie (Encadré), est une intervention délicate et précise, mais désormais bien codifiée (Figure $4 \mathrm{~A}$ ), reproductible et sûre. L'amélioration visuelle est parfois spectaculaire pour des cataractes avancées. En France, plus de 850000 yeux ont été opérés de cataractes au cours l'année 2019. Cela en fait l'acte chirurgical le plus pratiqué, toutes chirurgies confondues.

La vision peut récupérer en quelques jours, mais la cicatrisation se stabilise généralement dans le premier mois. La correction optique additionnelle peut être proposée une fois passé ce délai. Le résultat réfractif est d'autant plus prévisible que la réfraction initiale est proche de l'emmétropie, c'est-à-dire que l'image se focalise naturellement sur la rétine sans besoin de correction optique additionnelle.

II peut exister des complications, comme pour tout acte thérapeutique efficace. Elles sont résumées et expliquées dans la fiche d'information consensuelle éditée en ligne par la société française d'ophtalmologie $e^{3}$. Certaines sont peu rares mais sans conséquence significative. D'autres peuvent nécessiter des compléments chirurgicaux et/ou impacter la fonction visuelle finale. C'est le cas de l'œdème cornéen par insuffisance cellulaire endothéliale cornéenne (CEC).

\section{L'opération de la cataracte menace les cornées fragiles}

\section{Seuil à risque d'œdème de cornée}

On estime que le seuil de la dysfonction endothéliale se situe autour de 500 à $800 \mathrm{c} \varepsilon C / \mathrm{mm}^{2}$. En deçà de cette densité cellulaire, l'endothélium ne suffit plus à déturger la cornée correctement. II se produit alors un œdème du stroma cornéen. Un dérouillage visuel matinal qui se prolonge plus de 30 minutes, est le signe d'une fragilité de la fonction endothéliale significative. Une intervention chirurgicale de cataracte consomme nécessairement des CEC. On estime la perte de capital endothélial consécutive à une phacoémulsification normale non compliquée comme étant environ de 1 à $5 \%$. Lorsque les CEC sont altérées, non seulement leur densité est abaissée, mais leur propension à disparaître au cours d'une chirurgie augmente. La cornée peut alors ${ }^{3}$ http://www.sfo.asso.fr/files/files//FICHE-INFO-PATIENT_/O1A_Operation_de_la_
cataracte.pdf 


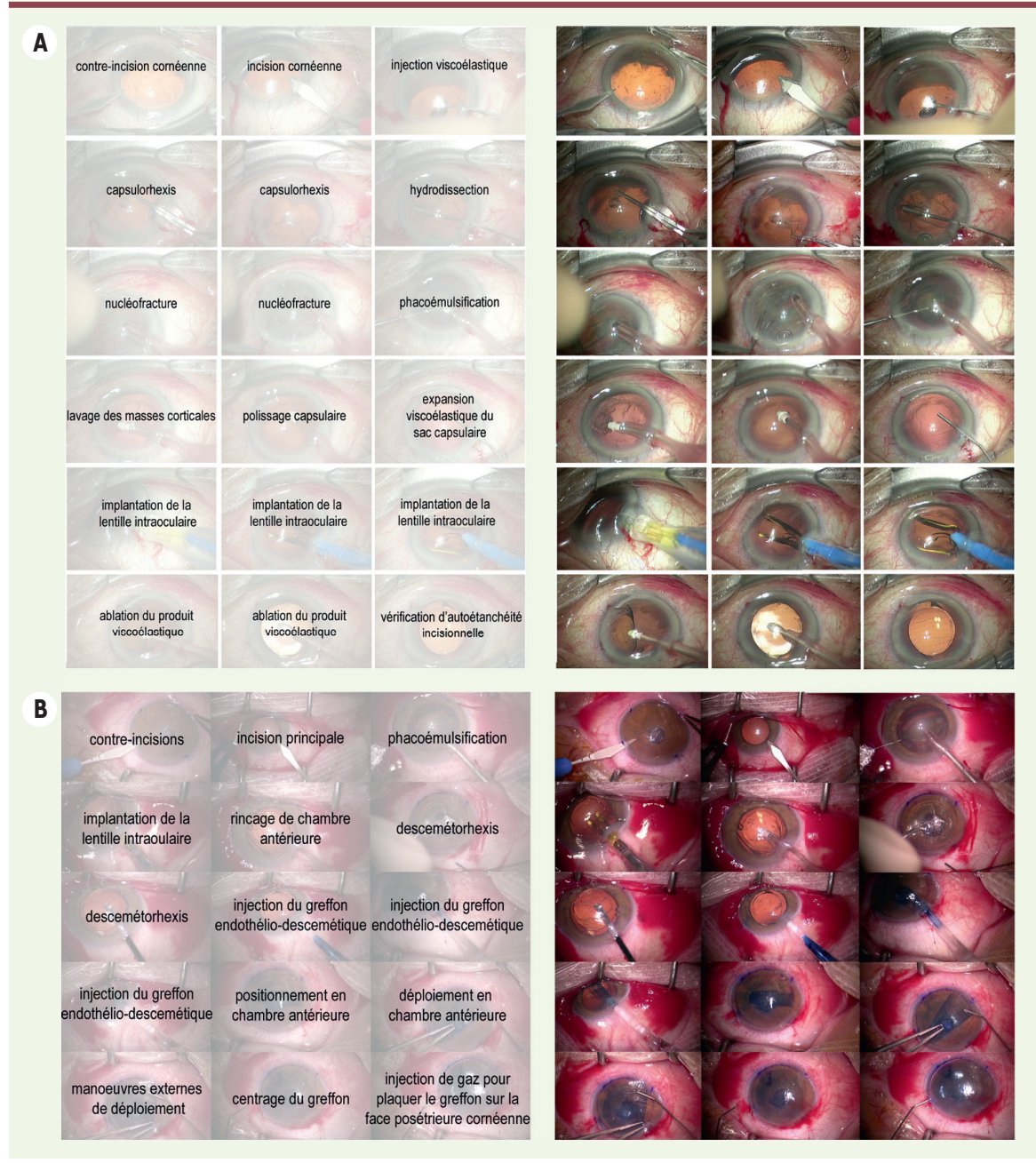

Figure 4. A. Les différentes étapes chirurgicales de l'intervention de cataracte par phacoémulsification sont stéréotypées (Encadré). La désinfection et l'anesthésie locale sont suivies techniquement par l'incision cornéenne, l'injection viscoélastique, le capsulorhexis, l'hydrodissection, la nucléofracture, le lavage des masses corticales, l'injection viscoélastique, l'implantation de la lentille intraoculaire, l'ablation du produit viscoélastique, la vérification d'autoétanchéité incisionnelle, l'antibioprophylaxie. B. On peut associer la phacoémulsification à la greffe de tissu endothélial, par exemple de type DMEK. Les étapes d'une DMEK (Descemet membrane endothelial keratoplasty) sont la préparation d'un greffon à partir d'une cornée de donneur sain, les incisions cornéennes, l'ablation de l'endothélium dysfonctionnel du patient sous air ou produit viscoélastique qu'il faudra alors rincer, l'injection du greffon endothélial, le déroulement de celui-ci et son placage sur la face interne cornéenne, endothélium vers la chambre antérieur. Toutes ces étapes s'aident de flux de liquide ou d'air pour ne pas manipuler directement le tissu trop fragile.

perdre plus de $20 \%$ de son capital endothélial. Ainsi, ceci s'applique à une cornée qui possèderait par exemple, une densité de CEC estimée autour de $1000 \mathrm{C} \varepsilon C / \mathrm{mm}^{2}$ avant une intervention de phacoémulsification. La chirurgie non compliquée correctement réalisée entraînera une diminution de la densité des CEC, qui atteindra alors environ $800 \mathrm{CEC/}$ $\mathrm{mm}^{2}$. La cornée sera alors placée après la chirurgie à son seuil de décompensation œdémateuse. L'épaisseur cornéenne préopératoire supérieure à 620-640 $\mu \mathrm{m}$ au centre de la cornée, semble également être un indicateur d'évolution péjorative [3].

\section{Stratégie de traitement}

Il serait déraisonnable d'être handicapé visuel en raison d'une cataracte lorsque sa chirurgie est accessible, même dans la situation d'une cornée fragile. En revanche, la stratégie de traitement est plus élaborée que lorsque la cornée est saine. Globalement, lorsque la gêne visuelle liée à la cataracte empêche d'effectuer les actes souhaités de la vie courante, intervenir est nécessaire. Avant, il faut considérer de s'abstenir. Si la gêne visuelle est plutôt d'origine cornéenne, alors il faut agir sans grand délai.

La stratégie thérapeutique privilégie les options allant des moins invasives, comme la surveillance, aux plus invasives, comme la greffe de cornée endothéliale (kératoplastie lamellaire endothéliale ou KLE). Au final, les options à considérer sont : la surveillance simple. En l'absence de gêne visuelle significative et tandis que la nécessité d'une kératoplastie peut s'imposer, il est préférable de s'abstenir temporairement de chirurgie ;

la cure de cataracte par phacoémulsification seule, suivie au besoin d'une KLE. En effet, il est toujours préférable d'épargner une greffe si cela est possible. Même si les chances sont faibles, l'option mérite d'être tentée ; la phacoémulsification combinée à la KLE (Figure 4B). Il est préférable de n'intervenir qu'une seule fois en chirurgie intraoculaire, en pratiquant d'emblée la KLE dès lors que la dysfonction endothéliale post-opératoire de cataracte semble inévitable ;

la KLE seule, suivie d'une phacoémulsification secondaire. Cette option est la seule possible quand la transparence cornéenne ne permet plus de pratiquer une phacoémulsification en toute sécurité. Elle est sous-optimale. Elle oblige à pratiquer une phacoémulsification après une précieuse $\mathrm{KL} \varepsilon$, au risque d'altérer cette dernière. 


\section{Remplacer l'endothélium}

Le remplacement endothélial nécessite actuellement une greffe de cornée, ou kératoplastie. La kératoplastie consiste à remplacer tout ou partie d'une cornée malade par une cornée saine prélevée chez un donneur décédé. Si toute la cornée est opacifiée et son collagène cicatriciel, il est possible de remplacer toute l'épaisseur cornéenne par une kératoplastie transfixiante $^{4}(K T)$. Son indication s'est raréfiée dans les systèmes de santé évolués. Les KLE lui sont désormais préférées en raison de leurs suites opératoires plus rapides, de leur meilleure réhabilitation visuelle, de leurs moindres complications et de leurs faibles taux de rejet. Deux techniques principales sensiblement équivalentes coexistent:

la ultra-thin descemet stripping assisted-endothelial keratoplasty (UT-DSAEK). Elle remplace l'endothélium malade par un greffon endothélial sain porté par une fine lamelle de son stroma postérieur [4] ; la Descemet membrane endothelial keratoplasty (DMEK) (Figure 4B). L'endothélium et sa membrane basale seuls sont prélevés et plaqués sur la face postérieure de la cornée malade dénudée de son endothélium dysfonctionnel [5].

Bien que déjà très abouties, il est toujours possible d'améliorer les solutions offertes aux patients à cornée fragile qui doivent se faire opérer de la cataracte. L'utilisation de produits viscoélastiques spécifiques et une gestuelle chirurgicale adaptée diminuent les risques cornéens de la phacoémulsification.

On entrevoit déjà les avancées prometteuses des équipes de recherche qui travaillent à améliorer encore le traitement de la dysfonction des CદC [6]. Les pistes les plus abouties explorent le raffinement des techniques chirurgicales et la bio-ingénierie avec l'induction de divisions des CEC associée à l'augmentation de leurs capacités cicatricielles et migratoires, la culture d'explants de CEC autologues ou allogéniques, ou l'injection seule en chambre antérieure des CEC. Toutes ces solutions et ces voies de recherche comptent sur le don du tissu cornéen et sur la chaîne de son prélèvement. C'est pourquoi il reste plus important que jamais d'informer les patients et il est toujours crucial que toute personne souhaitant être un donneur potentiel se détermine comme tel de son vivant. $\diamond$

When cataracts lead to a corneal transplant

\section{SUMMARY}

The eye has two converging lenses arranged in series: the cornea and the lens. They combine their powers. The image, which is naturally defocused ad infinitum, by crossing them successively, focuses on the retina to be seen clearly. Edema can cause the cornea to lose transparency while the clouding of lens leads to cataract. The loss of transparency of one or both lenses significantly affects the vision. Treating cataracts is a common practice. However, this can lead to the permanent loss of transparency of the cornea. A graft of the latter must then be carried out. How does this sometimes come about? $\diamond$

\section{LIENS D'INTÉRÊT}

L'auteur déclare n'avoir aucun lien d'intérêt concernant les données publiées dans cet article.

\section{RÉFÉRENCES}

1. Bonanno JA. Molecular mechanisms underlying the corneal endothelial pump. Exp Eye Res $2012 ; 95: 2-7$.

2. Liu YC, Wilkins M, Kim T, et al. Cataracts. Lancet 2017 ; $390: 600-12$.

3. Seitzman GD. Cataract surgery in Fuchs' dystrophy. Curr Opin Ophthalmol $2005 ; 16: 241-5$.

4. Busin M, Patel AK, Scorcia V, Ponzin D. Microkeratome-assisted preparation of ultrathin grafts for descemet stripping automated endothelial keratoplasty. Invest Ophthalmol Vis Sci 2012 ; 53 : 521-4.

5. Melles GR, Ong TS, Ververs B, van der Wees J. Descemet membrane endothelial keratoplasty (DMEK). Cornea $2006 ; 25$ : 987-90.

6. Mehta JS, Kocaba V, Soh YQ. The future of keratoplasty: cell-based therapy, regenerative medicine, bioengineering keratoplasty, gene therapy. Curr Opin Ophthalmol $2019 ; 30: 286-91$.

\section{TIRÉS À PART}

${ }^{4}$ De part en part.

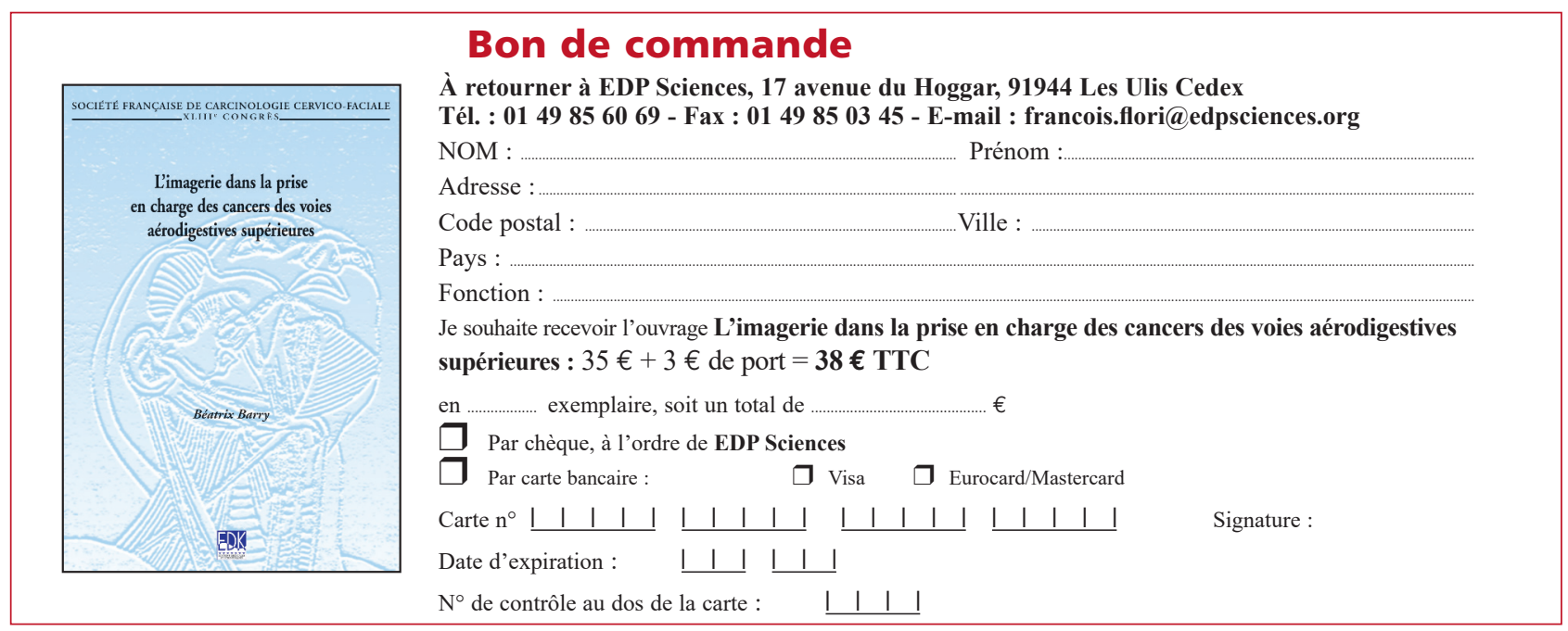

